\title{
Modelling Techniques Used in The Analysis of Stratified Thermal Energy Storage: A Review
}

\author{
Afzal Ahmed Soomro ${ }^{1}$, Ainul Akmar Mokhtar, Ali Akbar and Aijaz Abbasi \\ Department of Mechanical Engineering Universiti Teknologi PETRONAS
}

\begin{abstract}
Thermal energy storage plays an important role in the energy management and has got great attention for many decades; stratification is a key parameter to be responsible for the performance of the stratified thermal energy storage tank. In this paper detailed study of modelling techniques used to analyse thermal energy storage has been conducted. The division of literature has been made as numerical, analytical, and artificial neural network-based. Numerical modelling being very physical based and required more specific software's tools remain costly and computationally very complex at the same time it provides the detailed insights into the system, analytical model provide the exact solutions but need some assumptions which make the system unrealistic in some cases but is easy and flexible in terms of computational requirements, ANN though recently used modelling technique is a black box model which merely needs the data rather than any physical based complex calculations is attracting the scientific community.
\end{abstract}

\section{Introduction}

Energy storage technology is getting more attention day by day due to increasing need of the energy conservation. Due to the mismatch between the energy supply and energy demand, storage of energy becomes important for filling the gap between supply and demand during off-peak and on-peak periods. Various forms of energy are available to store e.g. mechanical, chemical and thermal [1]. Thermal energy is further divided into sensible and latent heat. In the case of latent heat, the material is supposed to change its form or phase change occurs. While for the sensible heat the temperature of the material changes without changing the phase of the material. In Sensible heat applications, water or rocks are widely used. Rock is used for air heating applications and water is suitable for the water cooling or heat purposes [2]. The water being very economical and easily available material has the property of stratification which separates the cold and hot bodies of water based on the density difference that is the key performance measure of the storage devices [2]. Stratification is the natural tendency of the water in which the hot and cold volumes of the water are being separated by virtue of the density difference and the thin separation layer is maintained between these two portions as shown in the Fig.1. The importance of temperature

\footnotetext{
${ }^{1}$ Corresponding author: afzals.09in09.muet@gmail.com
} 
stratification has been known since 1970 [3-5]. Stratification can be damaged or destratification in the tank can mostly be occur by four reasons i.e the heat loss to the environment, heat loss to the adjacent layers of the water, axial wall conduction and the mixing in the inlet jet of the water. These losses can be reduced by proper design of the tank and the diffusers used for the supply of the inlet jet. Since inception of the research in the realm of thermal energy storage various theoretical and experimental work has been carried out to identify the causes of destratification and how to improve the performance. The theoretical and experimental work has been carried out by using one-dimensional models and also two and three-dimensional models. Which were solved numerically as well as analytically. One-dimensional models being simple and less complex have been solved by both analytical and numerical methods, where as higher dimensional models being complicated have only been solve by numerical approach [6]. This paper review research conducted in modelling of stratified thermal energy storage tanks and categories them as numerical, analytical and neural network based.

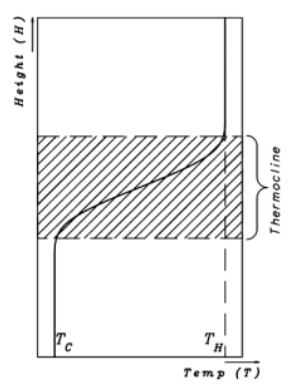

Fig.1 stratified thermal energy storage tank [7].

\subsection{Numerical modelling}

Numerical methods play an important role in determining the performance and the behaviour of the stratified thermal energy storage tank. Since, the temperature is a key factor which influences the performance and the behaviour of the thermal energy storage tank. It is necessary to predict the temperature profile curve within thermal energy storage tank. The temperature profile can be predicted by solving the physical equations which govern the system. They are mass, energy and momentum equations. Since, these equations are partial differential equations, that's why they need to be discretized to obtain the temperature inside the tank. Many models have been built to obtain the temperature profiles. Including MultiNode models and Plug-Flow models are widely used modelling techniques for modelling purpose. These models are based on the computer-based simulations and don't allow the manual calculations to simulate the models. The TRNSYS (Transient System Simulation Tool) software provide the types of models [8]. In multi-node modelling the tank is divided into a number of slabs as shown in Fig.2. 


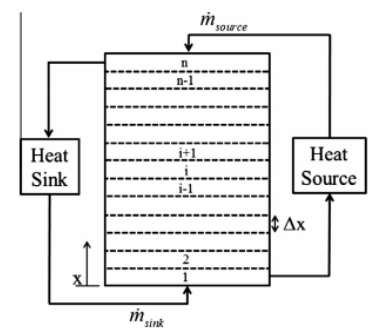

Fig.2. Multi-Node modelling of stratified thermal energy storage tank [9].

In this type of the modelling the slabs or nodes are supposed to interchange the heat to the adjacent slab $[8,10]$. The partial differential equations are discretized mostly by using finite difference methods(FDM) and the discretized equations are written on each slab or node in order to get the temperature distribution. Various researchers used this technique for obtaining the temperature profiles in the tank. Zurigat used the multi-node approach which was based on the buffer tank concept [11]. Joko also used the same buffer tank concept to simulate the performance of the stratified thermal energy storage tank of gas district cooling plant[12]. The reason for using the buffer tank concept was only to mitigate the numerical diffusion, which is the major problem in numerical analysis.

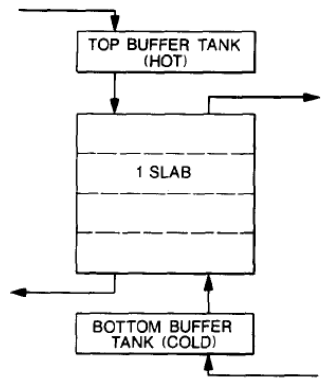

Fig. 3. Buffer tank concept of the TES model [11].

Another modelling technique is the plug flow model, in plug flow model the nodes or slabs of variable size, move up or down in a plug flow manner. In this model, the temperature distribution is simulated using the variable number of the slabs. These slabs cannot be controlled but vary depending upon the flow rate and volume of the tank. This model cannot solve the differential equations step by step but can be used in the built-in TRNSYS software [8]. This model gives better results than the multimode models because a smaller number of nodes are used, and the model becomes less complex. Hence, in modelling of the tank, the number of nodes or slabs plays an important role, to increase the number of nodes improves the degree of stratification but at the same time the computational complexity increases. Powel suggested the models which overcome this issue, they proposed adaptive grid model as shown in Fig.4. 


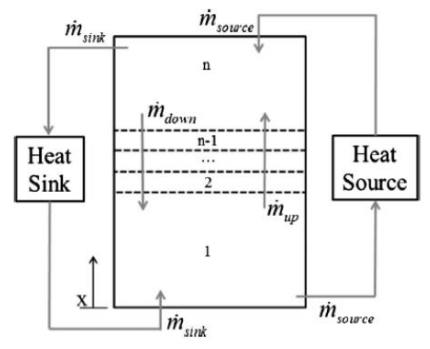

Fig. 4. Adaptive grid model [9].

This model is actually the combination of the multi-node and plug flow models. In this model more nodes have been created in the thermocline vicinity which is the most important area, the nodes upper and lower the thermocline region are considered as the singles nodes [9].

\subsection{Analytical methods}

Analytical methods are used in order to obtain the exact solutions rather than approximated solutions as in numerical modelling analytical models are based on many assumptions, which make the model less complex [1]. Many researchers have used the Laplace transformation for analytical modelling [4, 13-15]. Or using Green's function methodology [16]. And many researchers approximated the S-curves using Sigmoidal Doseresponse function (SDR) [17]. Cumulative distribution function (CDF) [18, 19]. and Fermi Dirac distribution [20]. Because the simulations based on numerical modelling done previously gave the solution in the form of S-curve. Analytical models are grid-less models which are computationally very less complex [21]. Yoo analytically solved the 1-D energy equation for adiabatic finite size stratified storage tank schematically shown in Fig.5. with fixed inlet temperature using Laplace transform techniques [13]. Joko used a SDR function to analytically analyse the stratified TES tank installed at Gas District Cooling (GDC) [17]. For this he used the temperature sensor data of the tank and analysed the data with the software package sigma plot. Joko also used the same analytical function to determine the performance parameters of hot stratified thermal energy storage tank and obtained the Scurves which provided the better predictions [22]. Bayon used Logistic cumulative distribution function (CDF) by replacing the normal cumulative distribution function [19]. Because it employs solving integrals which are avoided due to complexity.

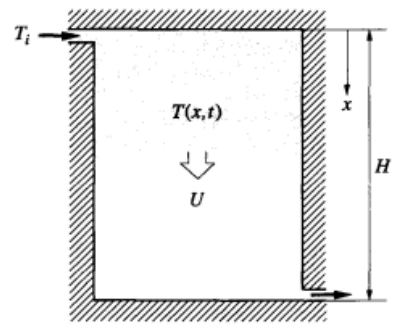

Fig.5. Schematic of thermal energy storage tank [13]. 


\subsection{Artificial neural networks}

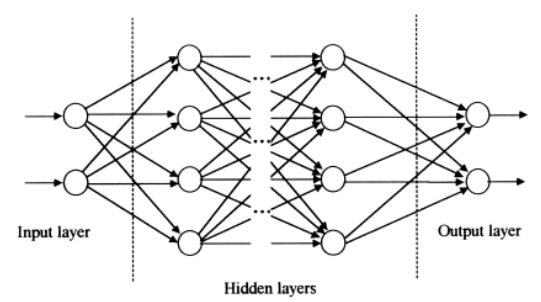

Fig.6. The standard model for ANN.

TES has extensively been simulated by the physical based modelling in numerical approach which solves the differential equations in the background which govern the physical laws these methods are computationally very complex and required special types of the software packages. Contrary to this approach, researchers are more focused on the black box modelling [23]. approaches due to their easy learning process and simplicity and low cost [24]. Artificial neural network (ANN) are non-linear systems for mapping which mimic the notion of the biological nervous systems of human being. An ANN encompasses many large simple processors linked with weighted connections. By analogy, the processing units are called neurons. Neural network models are basically different from the numerical methods to predict the future behaviour this approach has been used in multiple areas and provided the encouraging results. For example [25]. Although numerical and analytical approaches have been used in the modelling of TES systems, ANN modelling has only started to be applied [1]. Since, ANN is not physical modelling technique and is solely based on the available data which is used for inputs and outputs for the model the very important thing to be considered in the ANN modelling is the number of the data set used for the training purpose and also the number of the neurons used in the hidden layer in order to avoid the errors in prediction. Recently, an artificial neural network (ANN) scheme has been applied to the modelling of stratified TES tank [6]. P. Geczy-Vig used ANN tool in MATLAB to predict the temperature of the stratified layers in the solar thermal energy storage tank by training the model with 30943 data set of the input and output data as shown the Fig.7. where, T1(t-1),.., T8(t-1) is the average temperature data of each layer or slab of the storage tank during previous time step and $\mathrm{T} 1(\mathrm{t}), \ldots \mathrm{T} 8(\mathrm{t})$ are the temperatures of the layers in current time step used as the inputs for the model [26]. Though his model was merely based on the input-output data but gave better predictions than the physically based model previously built [27].

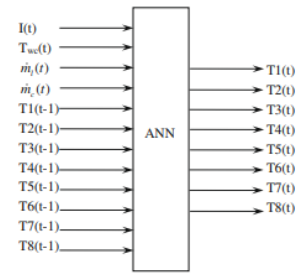

Fig.7. The layout of the applied ANN model [26].

Diez used ANN modelling to measure the temperatures in the hot solar stratified tank in static mode by using the input data of temperatures of 8 layers of the tank and the out put 
data as the temperature of the same layers after 10 minutes as shown in the Fig.8. [28]. by using MATLAB software, the results of the model gave satisfactory results and realized that the approach is easier and simpler as compared to the classical physical based modelling and has no technical data is required.

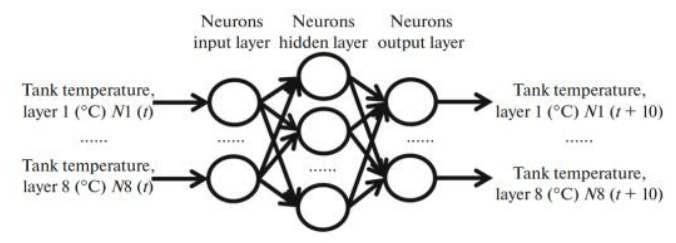

Fig.8. Structure of the ANN designed for prediction of stratification temperature at 10 minutes interval [28].

Lee used ANN tool based on neural network toolbox in MATLAB R2017a to predict the temperature profile curve in the stratified TES tank and determine that how the temperature changes in the bottom as well as all the other layers or slabs of the tank. For training purpose, the data was obtained from the TES tank installed at Tokyo. For training 51913 data set were used. The inputs $Q^{t-1}, Q^{t}, T_{20}^{t-1}$ and for output $T_{20}^{t}$ where used, where, $Q^{t-1}, Q^{t}, T_{20}^{t-1}$ are the remaining heat and outlet temperature i.e temperature of the 20 th layer of the previous time step, predicted remaining heat of the current time step and temperature in the 20th layer of previous time step respectively and $T_{20}^{t}$ is the temperature of the outlet temperature of the tank [24].

\section{Conclusion}

Stratified thermal energy storage plays an important role in filling the gap between the demand and supply, therefore to measure the performance of the TES tank is also very important. Performance of the TES tank has been measured by analysing the temperature profiles within the tank, to predict the temperature profiles numerical methods based on the physical laws, analytical methods based on assumptions, and recently artificial neural network based on data-driven approach has been adopted. Numerical methods are actually more physical based and provided the detailed modelling information, but it requires high computer power, more simulation time and require special types of software, analytical methods also have been extensively used due to their less complexity and now recently artificial neural networks have attracted many researchers to work in order to get rapid and accurate solutions without using extensive physical based modelling.

\section{Reference}

1. I. Dincer and M. Rosen, Thermal energy storage: systems and applications: John Wiley \& Sons, (2002)

2. J. A. Duffie and W. A. Beckman, John Willey \& Sons, New York, (1980)

3. J. van Berkel, C. C. Rindt, and A. A. van Steenhoven, Solar Energy, 67, 65-78 (1999)

4. A. Cabelli, Sol. Energy. 19, 45-54 (1977)

5. K. Hollands and M. Lightstone, Sol. Energy. 43, 97-105 (1989)

6. H. Njoku, O. Ekechukwu, and S. Onyegegbu, Heat Mass Transfer, 50, 1017-1030 (2014)

7. A. Karim, A. Burnett, and S. Fawzia, Energies, 11, Article number-1049 (2018) 
8. J. A. Duffie and W. A. Beckman, Solar engineering of thermal processes: John Wiley \& Sons, 2013.

9. K. M. Powell and T. F. Edgar, Energy Convers. Manage. 76, 865-873 (2013)

10. E. M. Kleinbach, "Performance study of one-dimensional models for stratified thermal storage tank," University of Wisconsin--Madison, (1990)

11. F. Oppel, A. Ghajar, and P. Moretti, Appl. Energy, 23, 205-224 (1986)

12. J. Waluyo, in AIP Conference Proceedings, 030002 (2016)

13. H. Yoo and E.-T. Pak, Solar Energy, 56, 315-322 (1996)

14. W. Karaki, J. T. Van Lew, P. Li, C. L. Chan, and J. Stephens, in ASME 2010 4th International Conference on Energy Sustainability, 725-734 (2010)

15. O. Dumont, C. Carmo, R. Dickes, E Georges, S Quoilin, V. Lemort, CLIMA (2016).

16. M. W. Jack and J. Wrobel, Appl. Therm. Eng. 29, 2344-2349 (2009)

17. J. Waluyo, M. A. A. Majid, and M. Amin, Int J Mech Mechatron Eng, 1, 7-12 (2010)

18. R. Bayón, E. Rivas, and E. Rojas, Energy Procedia, 49, 725-734 (2014)

19. R. Bayón and E. Rojas, Int. J. Heat Mass Transfer. 68, 641-648 (2014)

20. J. D. Chung and Y. Shin, Sol. Energy. 85, 3010-3016 (2011)

21. C. Unrau, Numerical Investigation of One-Dimensional Storage Tank Models and the Development of Analytical Modelling Techniques (McMaster University, CA, 2017).

22. J. Waluyo, in Engineering Seminar (InAES), International Annual, 218-222 (2016).

23. R. Kicsiny, Renew. Energy, 125, 857-865 (2018)

24. D. Lee, R. Ooka, S. Ikeda, and W. Choi, COBEE 2018, 333-338 (2018).

25. D. D. Massie, Int. J. Therm.Sci. 41, 1121-1129 (2002)

26. P. Géczy-Víg and I. Farkas, Sol. Energy. 84, 801-806 (2010)

27. P. Ge'czy-V1'g, Farkas, I, Proceedings of the 11th International Conference on Solar Energy in High Latitudes, NorthSun 2007, Riga, Latvia, (2007)

28. F. Diez, L. Navas, and A. Martínez, Appl. Sol. Energy. 52, 183-192 (2016) 